\title{
Test of Conformal Invariance in One-Dimensional Quantum Liquid with Long-Range Interaction
}

\author{
Rudolf A. Römer and Bill Sutherland \\ Physics Department, University of Utah, Salt Lake City, UT 84112 \\ (Received )
}

\begin{abstract}
We numerically study the momentum distribution of one-dimensional Bose and Fermi systems with long-range interaction $g / r^{2}$ for the "special" values $g=-\frac{1}{2}, 0,4$, singled out by random matrix theory. The critical exponents are shown to be independent of density and in excellent agreement with estimates obtained from $c=1$ conformal finite-size scaling analysis.
\end{abstract}

Typeset Using REVTEX 


\section{INTRODUCTION}

In one-dimensional (1D) quantum systems it has been argued that the existence of a critical point at zero temperature implies asymptotic conformal invariance at large distances [19,3]. This invariance powerfully constrains the possible behavior of a given critical theory, parametrizing universality classes by the conformal anomaly $c$, the value of the central charge of the underlying Virasoro algebra [3]. For $c<1$, it has been shown by Friedan, Qui and Shenker that unitarity (reflection positivity) restricts those values of $c$ to be quantized [9]. The critical exponents are given by the Kac-formula and the correlation functions can be determined. Realizations of this scheme are the Ising $\left(c=\frac{1}{2}\right)$, tricritical Ising $\left(c=\frac{7}{10}\right)$, three-state Potts $\left(c=\frac{4}{5}\right)$, tricritical Potts $\left(c=\frac{6}{7}\right)$, and other models [5,7]. In addition, a complete classification can also be given for $c \geq 1$ if a continuous symmetry is assumed; e.g. for $U(1)$ we get the Gaussian model $(c=1)$, describing a variety of models such as the Coulomb gas, XY-model, $s=\frac{1}{2}$ antiferromagnet and more [5, 6].

The basic assumptions leading to conformal invariance of a model may be summarized as scale invariance, rotational and translational invariance and short-range interactions. If any of the above properties do not hold, the conformal symmetry will in general not be obeyed either. In addition, it should be noted, that there is no rigorous proof of conformal invariance at a critical point, just as for scale invariance. Instead conformal invariance should be regarded as a principle, whose consequences can then be compared with exact and numerical results on specific models [5]. In the case of long-range interactions, an explicit counterexample may be given: Thouless [24 showed that certain long-ranged 1D classical systems may have a critical point. Now, since any general coordinate transformation in one dimension is conformal, this would imply that all correlation functions are constant, which is certainly not true.

Recently, however, a number of groups have independently applied conformal arguments to long-range models such as the periodic $g / r^{2}$ models 13 15, 18. They were able to compute the conformal anomaly and the conformal scaling dimensions, as well as various correlation exponents for Bose and Fermi statistics as functions of the interaction strength $g$. Their predictions agree with the exact result for the case of $g=0$, that is, free fermions and hard core bosons. For less trivial interaction strength, no independent verification of these results exists so far.

The periodic $g / r^{2}$ model has been solved by means of the Bethe Ansatz by one of us several years ago [22]. As it turns out, for the three "special" values $g=-\frac{1}{2}, 0,4$ of the interaction strength, the ground state wave function of this model can be viewed as a distribution function for the eigenvalues of a random matrix from an appropriate ensemble. Application of theorems from random matrix theory then allows the derivation of explicit formulae for the ground state correlation functions [23].

In this paper, we will present a high-precision study of the critical exponents for the momentum distribution, which we will then compare to the results obtained by the conformal conjecture. In section II, we will outline the arguments of conformal invariance leading to the predictions for the critical exponents. Section III introduces the $g / r^{2}$ model and its ground state correlations in more detail. We present the results of our numerical study in section $\amalg$, where in addition we present an unusual scaling law. 


\section{DERIVATION OF CRITICAL EXPONENTS}

Finite-size scaling analysis of classical two-dimensional statistical systems at criticality may be used to obtain the value of the conformal anomaly $c$ and the scaling dimensions $x_{n}$. For short-range interactions that give rise to a linear dispersion relation, i.e. gapless excitations, the following formulae hold [4]: for a strip of width $L \rightarrow \infty$ and periodic boundary conditions,

$$
\begin{aligned}
E_{0} & \sim \epsilon_{0} L-\frac{\pi}{6} \frac{c v}{L}, \\
E_{n}-E_{0} & \sim 2 \pi \frac{x_{n} v}{L} .
\end{aligned}
$$

Here $E_{n}$ is the eigenvalue of the Hamiltonian; $E_{0}$ is the ground state energy; $\epsilon_{0}$ is the ground state energy density in the thermodynamic limit, and $v$ is the velocity of the elementary excitations. Since the partition function of a classical system of finite width and periodic boundary conditions may be viewed as the Feynman path integral for an infinitely long quantum chain at finite temperature, these results apply to $1 \mathrm{D}$ quantum systems as well [1, 2].

We now wish to evaluate the scaling dimensions for the 1D Bose and Fermi systems interacting via the long-range interaction of the periodic $g / r^{2}$ model. This model has been solved by the asymptotic Bethe Ansatz in [22], and it has been shown that the energy spectrum may be written as

$$
E_{\left\{I_{j}\right\}}=\sum_{j=1}^{M}\left[\frac{2 \pi}{L} I_{j}+\frac{\pi \lambda}{L}(2 j-M-1)\right]^{2},
$$

where $\left\{I_{j}\right\}$ denotes the set of ordered (free boson) quantum numbers of a particular state; $M$ is the number of particles; $L$ is the length of the ring and $2 \lambda(\lambda-1)=g$. The ground state energy $E_{0}$ corresponds to the state with quantum numbers $I_{j}=0$ for all $j=1, \ldots, M$. In addition, the model has a sound type excitation spectrum, and for $\lambda=\frac{1}{2}, 1,2$ all correlation functions may be calculated. We will elaborate on this last fact in more detail in the next section.

Let us now calculate the finite-size corrections to the excited states. We start with the Bose system first. An excitation corresponding to a momentum transfer $P=0$ is given by changing the number of particles $M$ to $M+\Delta M$. By (3), the excitation energy is given as

$$
\begin{aligned}
E(M+\Delta M)-E(M) & =\frac{\pi^{2} \lambda^{2}}{L^{2}}\left\{\sum_{j=1}^{M+\Delta M}[2 j-(M+\Delta M)-1]^{2}-\sum_{j=1}^{M}[2 j-M-1]^{2}\right\} \\
& \simeq \pi^{2} \lambda^{2} d^{2} \Delta M+\frac{1}{L} \pi^{2} \lambda^{2} d \Delta M^{2} \\
& =\mu \Delta M+\frac{2 \pi v}{L} \frac{\lambda}{4} \Delta M^{2}
\end{aligned}
$$

Here we used the formulae for the chemical potential $\mu=\pi^{2} \lambda^{2} d^{2}$ and the sound velocity $v=2 \pi \lambda d$, as obtained in 22 .

Let us define the Fermi momentum as $k_{F}=\pi d$. Then for Fermi and Bose systems and fixed particle number $M$, taking $\Delta D$ particles from the "left" Fermi momentum to the 
"right" Fermi momentum — or equivalently, by translating the filled Fermi sea in momentum space - results in another type of low-lying excitation, i.e.

$$
\begin{aligned}
E(M ; \Delta D)-E(M ; 0) & =\sum_{j=1}^{M}\left\{\left[\frac{2 \pi}{L} \Delta D+\frac{\pi \lambda}{L}(2 j-M-1)\right]^{2}-\left[\frac{\pi \lambda}{L}(2 j-M-1)\right]^{2}\right\} \\
& =\frac{2 \pi v}{L} \frac{1}{\lambda} \Delta D^{2}
\end{aligned}
$$

The momentum of this excitation is $P=2 k_{F} \Delta D$.

For fermions, the same reasoning applies. However, the quantum numbers $I_{j}$ are restricted to integers now and we have to follow the selection rule

$$
\Delta D=\frac{\Delta M}{2}(\bmod 1)
$$

By combining (4) and (5), we obtain the scaling dimensions for the above excitations as

$$
x(\Delta M ; \Delta D)=\frac{\lambda}{4} \Delta M^{2}+\frac{1}{\lambda} \Delta D^{2} .
$$

This expression is valid for both Bose and Fermi statistics modulo the selection rule (6).

We have not calculated the effect of particle-hole excitations on $x$. Although we do not need the resulting additional terms for the calculation of the long-distance behavior of the correlations, we remark, however, that in order to correctly read off the right and left conformal weights that characterize the underlying Virasoro algebra, they cannot be ignored 3,4, $4,18,14$.

Before we continue with our derivation of the critical exponents of the momentum distribution, let us pause and first calculate the critical anomaly $c$ for the present model. From (3) it follows that

$$
E_{0} \equiv E_{\{0\}}=\epsilon_{0} L-\frac{1}{3} \pi^{2} \lambda^{2} d / L
$$

Using the above expression for the sound velocity $v$, we may rewrite the $L^{-1}$ correction term as $-\frac{\pi v}{6 L} \lambda$. Comparison with (2) yields $c=\lambda$. This is a peculiar result in as much as the critical anomaly varies continuously with the interaction strength $\lambda$. We could instead use the low-temperature expansion of the free energy as given in [22] and the formula for the critical anomaly as in [1,2], giving

$$
F(T) \simeq F(T=0)-\frac{\pi T^{2}}{6 v}
$$

and therefore $c=1$. Thus the two expressions for $c$ are in contradiction. We will discuss this point in more detail in section $\mathrm{D}$.

Let us finally investigate the long-distance behavior of the correlation functions. Let the pair $(\Delta M ; \Delta D)$ describe an excitation where the particle number is changed by $\Delta M$, and $\Delta D$ particles are transported round the ring. If we then denote by $\phi^{\dagger}(\phi)$ the creation (annihilation) operator of this excitation, the corresponding leading term of the equal-time correlator has the form 


$$
\left\langle\phi^{\dagger}(r) \phi(0)\right\rangle \propto \cos (P r) r^{-2 x(\Delta M ; \Delta D)}
$$

where $P$ is the associated momentum carried by the excitation [5]. Therefore the correlation of the Bose field operator has the form

$$
\left\langle\phi_{B}^{\dagger}(r) \phi_{B}(0)\right\rangle_{\lambda} \propto r^{-\beta_{B, \lambda}}
$$

where the corresponding excitation is given by $(1 ; 0)$, and consequently by (7) $\beta_{B, \lambda}=\lambda / 2$. Due to the selection rule (6) for fermions, the corresponding excitation of the correlation

$$
\left\langle\phi_{F}^{\dagger}(r) \phi_{F}(0)\right\rangle_{\lambda} \propto \cos \left(k_{F} r\right) r^{-\beta_{F, \lambda}}
$$

is given as $\left(1, \frac{1}{2}\right)$ and thus $\beta_{F, \lambda}=(\lambda+1 / \lambda) / 2$.

Taking the Fourier transform of the long-distance expressions (10) and (11), we get the momentum distribution function $n(k)$. The long-distance behavior now translates into the behavior at long-wavelength or small wave numbers, and thus for the Bose momentum distribution near the origin,

$$
n_{B}(k) \propto|k|^{\alpha_{B, \lambda}}=|k|^{\beta_{B, \lambda}-1} .
$$

For the Fermi case, we have correspondingly for the momentum distribution near $k_{F}$,

$$
n_{F}(k) \propto\left|k-k_{F}\right|^{\alpha_{F, \lambda}} \operatorname{sgn}\left(k-k_{F}\right)=\left|k-k_{F}\right|^{\beta_{F, \lambda}-1} \operatorname{sgn}\left(k-k_{F}\right)
$$

In Fig. 1 w we show the behavior of the critical exponents as functions of $\lambda$. We emphasize that the exponents do not depend on the particle density $d$, but on $\lambda$ only.

Our above derivation of the critical exponents follows largely the article of Kawakami and Yang [14]. Other derivations have been given by Mironov and Zabrodin, who obtained the correlation exponents for a wider class of 1D quantum models with pairwise interaction [18], and by Kolomeisky [15], who applied methods of two-dimensional elasticity theory to arrive at corresponding results.

\section{CORRELATIONS IN THE SUTHERLAND MODEL}

The ground state wave function for the periodic $g / r^{2}$ model with $M$ particles, either bosons or fermions, interacting on a one-dimensional ring of circumference of length $L$, is of the Jastrow product form and given by 22,23.

$$
\begin{aligned}
\Psi_{\lambda} & =\left(\frac{\Gamma(1+\lambda)^{M}}{L^{M} \Gamma(1+\lambda M)}\right)^{\frac{1}{2}} \prod_{1 \leq j<k \leq M}\left|2 \sin \left[\pi\left(x_{k}-x_{j}\right) / L\right]\right|{ }^{\lambda} S \\
& \equiv C_{\lambda, M} \prod_{1 \leq j<k \leq M} \psi_{\lambda}\left[2 \pi\left(x_{k}-x_{j}\right) / L\right] .
\end{aligned}
$$

The factor $S$ is equal to 1 for bosons, while for fermions $S=(-1)^{P}$ is the parity of the particle ordering permutation $P, x_{P 1}<\cdots<x_{P M}$. The periodicity in $L$ is assured if $M$ is odd. $\psi_{\lambda}(\theta)=|2 \sin (\theta / 2)|^{\lambda}$ for bosons and $\psi_{\lambda}(\theta)=|2 \sin (\theta / 2)|^{\lambda} \operatorname{sgn}(\theta)$ for fermions, the normalization constant is given as 


$$
C_{\lambda, M}^{2}=\frac{\Gamma(1+\lambda)^{M}}{L^{M} \Gamma(1+\lambda M)}
$$

As has been noted in [22], the square of $\Psi_{\lambda}$ for the "special" values $g=-\frac{1}{2}, 0,4$ - or $\lambda=\frac{1}{2}, 1,2$ - may be recognized as being identical to the joint probability density function for the eigenvalues of matrices from Dyson's circular ensemble [8], $\lambda=\frac{1}{2}, 1$ and 2 corresponding to orthogonal, unitary and symplectic ensembles, respectively.

Results from the theory of random matrices now enable the calculation of various correlation functions [17]. We will use the following result: For $u(\theta)=u(-\theta)=u(2 \pi+\theta)$, arbitrary otherwise, and the special values of $\lambda$,

$$
\left\langle\prod_{j=1}^{M} u\left(\theta_{j}\right)\right\rangle_{\lambda}=\operatorname{det}\left[F_{p q}\right]
$$

where for $\lambda=1$,

$$
\begin{gathered}
F_{p q}=\frac{1}{2 \pi} \int_{-\pi}^{\pi} \mathrm{d} \theta u(\theta) \cos (p-q) \theta, \\
p, q=\frac{1}{2}, \frac{3}{2}, \ldots, M-\frac{1}{2}
\end{gathered}
$$

for $\lambda=2$,

$$
\begin{gathered}
F_{p q}=\frac{1}{2 \pi p} \int_{-\pi}^{\pi} \mathrm{d} \theta u(\theta)[p \cos (p \theta) \cos (q \theta)+q \sin (p \theta) \sin (q \theta)], \\
p, q=\frac{1}{2}, \frac{3}{2}, \ldots, M-\frac{1}{2}
\end{gathered}
$$

finally for $\lambda=\frac{1}{2}$,

$$
\begin{gathered}
F_{p q}=\frac{p}{4 \pi} \int_{-\pi}^{\pi} \mathrm{d} \theta u(\theta) \int_{-\pi}^{\pi} \mathrm{d} \phi u(\phi) u(\phi) \operatorname{sgn}(\theta-\phi)[\cos (p \phi) \sin (q \theta)-\cos (p \theta) \sin (q \phi)], \\
p, q=\frac{1}{2}, \frac{3}{2}, \ldots, \frac{M}{2}-\frac{1}{2}
\end{gathered}
$$

and we assume $M$ to be even for all three cases. Thus, $F_{p q}$ is an $M \times M$ matrix for $\lambda=1,2$ and an $\frac{M}{2} \times \frac{M}{2}$ matrix for $\lambda=\frac{1}{2}$.

The one-particle density matrix is defined as

$$
\rho\left(x-x^{\prime}\right)=M \prod_{j=1}^{M-1} \int_{0}^{L} \mathrm{~d} x_{j} \Psi_{\lambda}\left(x_{1}, \ldots, x_{M-1}, x\right) \Psi_{\lambda}\left(x_{1}, \ldots, x_{M-1}, x^{\prime}\right),
$$

which with the help of (14) may be written as

$$
\begin{aligned}
\rho\left(x-x^{\prime}\right) & =M \frac{C_{\lambda, M}^{2}}{C_{\lambda, M-1}^{2}}\left\langle\prod_{j=1}^{M-1} \psi_{\lambda}\left(\theta_{j}-2 \pi x / L\right) \psi_{\lambda}\left(\theta_{j}-2 \pi x^{\prime} / L\right)\right\rangle_{\lambda, M-1} \\
& =\frac{M}{L} \frac{\Gamma(1+\lambda) \Gamma(1+\lambda(M-1))}{\Gamma(1+\lambda M)} \operatorname{det}\left[F_{p q}\right] .
\end{aligned}
$$


In the last formula, we use the appropriate matrix $F_{p q}$ with $M$ replaced by $M-1$. By the periodicity condition on the wave function, we restrict $M$ to be odd. Thus, $M-1$ is even and our above formulae are consistent. The normalization of $\rho$ has been chosen such that $\rho(0)=M / L=d$. The momentum distribution $n(k)$ is defined as the Fourier transform of $\rho$, i.e.

$$
n(k)=\int_{-\infty}^{\infty} \mathrm{d} r \rho(r) e^{-i k r}
$$

and the normalization is such that

$$
2 \pi d=\int_{-\infty}^{\infty} \mathrm{d} k n(k)
$$

The $\lambda=1$ case corresponds to free fermions or hard core bosons. Free fermions are well understood, and we find

$$
\rho(r)=\frac{\sin (\pi d r)}{\pi r}, \quad n(k)= \begin{cases}\frac{1}{2 \pi}, & |k|<\pi d \\ 0, & |k|>\pi d\end{cases}
$$

Thus, $\alpha_{F, 1}=0$ in agreement with (13). Hard core bosons have been treated extensively in the literature [11,20,16]. There is no condensation into a single momentum state, and it is proven that $n(k)$ diverges as $|k|^{-\frac{1}{2}}$ at the origin. Again, this is in agreement with the

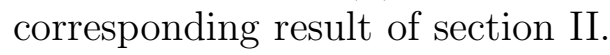

For $\lambda=2$, the matrix $F_{p q}$ has a particularly simple Toeplitz form, which allows the explicit calculation of $\rho$ [22],

$$
\begin{gathered}
\rho(r)=\frac{\operatorname{Si}(2 \pi d r)}{2 \pi r}, \quad \operatorname{Si}(r) \equiv \int_{0}^{r} \mathrm{~d} x \frac{\sin (x)}{x} \\
n(k)= \begin{cases}\frac{\ln (2 \pi d /|k|)}{4 \pi}, & |k| \leq 2 \pi d \\
0, & |k| \geq 2 \pi d\end{cases}
\end{gathered}
$$

Thus, the conformal result $\alpha_{B, 2}=0$ agrees again; although, the logarithmic behavior is not universal and so does not follow from the finite-size analysis.

This leaves the remaining three cases - bosons with $\lambda=\frac{1}{2}$, and fermions with $\lambda=\frac{1}{2}$ and $\lambda=2$ - to be investigated numerically.

Let us now study the restriction of the above family of wave functions to a lattice. The size of the systems $L$ is now an integer $N$, and the coordinates of the particles are restricted to integers $x_{j}=1,2, \ldots, N$. The wave functions are nearly as before; the only substantial change is the normalization constants $C_{\lambda, M}$. For the special values of $\lambda$, the new $C_{\lambda, M}^{\prime}$ are known [23]. The one-particle density matrix $\rho(r)$ and the corresponding momentum distribution function $n(k)$ for the lattice case may still be calculated as before, if we replace the integrals by appropriate sums over lattice sites.

Note, however, that whereas the density $d=M / L$ in the continuum case entered trivially into all expressions as a length scale, the new density $d^{\prime}=M / N$ in the discrete case is dimensionless and will thus enter all expressions in an essential way. Indeed, the previous continuum results are the zero-density limit of the discrete case. We showed in section II that 
the critical exponents of the momentum distribution, as obtained from conformal analysis, should be independent of density. This prediction may therefore be verified by studying both continuum and lattice cases.

However, we want to emphasize that whereas the wave functions for the continuous case describe the ground state of the periodic $g / r^{2}$ model, there need not be any corresponding Hamiltonian for the wave functions of the lattice gas. For $\lambda=1$, we of course have either hard core bosons of free fermions; while for $\lambda=2$ bosons, we have the Haldane-Shastry model [10,21, 12] of a Heisenberg magnet with $1 / r^{2}$ exchange. However, for the remaining three cases - precisely the cases we are to evaluate numerically - it is not clear to what specific model our results belong to in the discrete case. From the conformal viewpoint, of course, the results represent a universality class of models.

Finally, the lattice gas can be shown to exhibit a particle-hole symmetry, such that we only need to consider $d \leq \frac{1}{2}$. In addition, at half-filling, Fermi statistics ensure $\rho(r)=0$ for $r \neq 0$ or even and therefore for the momentum distribution, the symmetry $n(k)+n(k+\pi)=1$ holds.

\section{RESULTS}

Using the results obtained in section [II, we calculate the one-particle density matrix $\rho\left(x-x^{\prime}\right)$, or by translation invariance $\rho(r)$, with the help of (15) in terms of the appropriate matrix $F_{p q}$. Taking the Fourier transform, we compute the momentum distribution $n(k)$, which, for fixed $L$ and $N$, turns out to have a slightly different quantitative behavior for the continuum and discrete cases. In Fig. A and 3, we show $n(k)$ corresponding to the discrete case at the special values $\lambda=\frac{1}{2}, 1,2$ and half-filling, for bosons and fermions, respectively.

The extraction of the critical exponents $\alpha_{B, \lambda}$ for Bose and $\alpha_{F, \lambda}$ for Fermi statistics is complicated by finite-size effects. The interchange of the $L \rightarrow \infty$ and $k \rightarrow 0\left(k \rightarrow k_{F}\right)$ limits is non-trivial, and the correct order $-L \rightarrow \infty$ limit first - is hard to achieve. In addition, we know from (16) that the momentum distribution for the bosonic $\lambda=2$ case has a logarithmic behavior, which is not predicted by the conformal analysis, and the possible appearance of this or some other finite-size effect for the remaining cases may further obscure the extraction of the correlation exponents.

However, a careful analysis of our data reveals that the one-particle density matrix $\rho$ obeys an unusual scaling law. For different lattice sizes $L$, a limiting curve $\rho_{\infty}$ may be constructed as

$$
\rho_{\infty}(r)=\frac{1}{L^{\beta_{\lambda}}} \rho_{L}(r / L)
$$

both for Bose and Fermi statistics. Therefore, the limit $L \rightarrow \infty$ of $\rho_{L}(r)$ may be taken at any point $r$, and we can directly extract the asymptotic value of the critical exponents of the momentum distribution by studying the long-distance behavior, i.e. $\rho(L / 2)$. In Fig. 4 we show the remarkably good convergence of our estimates for the critical exponents as function of the inverse length $1 / L$ of the ring for the two $\lambda=\frac{1}{2}$ cases and the fermionic $\lambda=2$ case.

The scaled one-particle density function exhibits another quite remarkable property. In Fig.'s 5 and 6, we have plotted $\rho_{\infty}$ for bosons and fermions and normalized it so that its 
value at the end points equals 1 . Although, as we remarked above, $n(k)$ is different for the discrete and the continuous case, $\rho_{\infty}$ is identical for both cases up to the accuracy of our calculation and thus independent of density. In addition, the curves corresponding to $\lambda=\frac{1}{2}$ and $\lambda=2$ in Fig. 6 lie on top of each other. This seems to indicate that $\rho_{\infty}$ is the same for identical values of the critical exponent $\alpha$. This is further supported by the fact that the bosonic $\lambda=2$ curve, corresponding to $\alpha=0$, is the same as the fermionic $\lambda=1$ case, corresponding to $\alpha=0$, too. We will discuss the implications of these findings in more detail in the next section.

We have calculated the critical exponents, both for the bosonic and fermionic cases and $\lambda=1,2$, for up to $M=641$ particles on $L=1282$ sites. Since the scaling curves are identical for discrete and continuum cases, so are the exponents. An independent estimate from $n(k)$ directly confirms this. For $\lambda=\frac{1}{2}$, the continuum formulae require the computation of double integrals of transcendental functions rather than orthogonal functions, and so do not reduce to the calculation of simple Kronecker $\delta$ 's. Therefore, calculations could be done only up to $M=41$ particles at half-filling. The discrete expressions require less computational effort and data for up to $M=161$ for the bosonic case, and $M=321$ for the fermion case have been calculated. Again, the results indicate that the critical exponents are the same, independent of whether the continuum or the discrete formulae are used. Since the discrete case is the low-density limit of the continuous case, we have therefore established that up to our numerical accuracy, the critical exponents $\alpha_{F, \lambda}$ and $\alpha_{B, \lambda}$ are independent of the density $d$ as predicted by the conformal arguments.

Let us now compare the values of our numerical estimates with the predictions of section III for the critical exponents. Preliminary estimates for small particle numbers indicated a disagreement with the predictions of the conformal analysis for the bosonic $\lambda=\frac{1}{2}$ case [23]. In table \&, we show that our present study shows excellent agreement of conformal and numerical results; the estimates for the exactly known results are included as a check on the numerical analysis.

Finally, we note that we could not find any logarithmic behavior except - as predicted — for the bosonic $\lambda=2$ case. However, there do seem to be substantial finite-size effects.

\section{DISCUSSION}

In the preceding sections, we have reviewed arguments of conformal finite-size scaling analysis leading to predictions for the critical exponents of the momentum distribution in the $1 \mathrm{D}$ quantum liquid with long-range interaction $g / r^{2}$. Both for bosonic and fermionic systems and at the special values $g=-\frac{1}{2}, 0,4$ for the interaction strength, we then proceeded to calculate the same exponents by use of explicit formulae as given by random matrix theory. For non-trivial cases, no analytic solutions exist and we calculated the exponents numerically. This is most conveniently done by discretizing the continuous problem to a lattice problem. The continuum results are then recovered by taking the low-density limit of the lattice system. Our results show that the values of the correlation exponents are the same for lattice and continuum case, thus independent of density. This verifies the corresponding prediction of conformal field theory. In addition, the numerical estimates of $\alpha_{B}, \alpha_{F}$ are in excellent agreement with the conformal predictions. 
The numerical analysis was helped by the appearance of the unusual scaling law (17). As it turns out, the scaled and properly normalized one-particle density function $\rho_{\infty}$ may be parametrized by the critical exponent $\alpha$ only, independently of statistics and interaction strength $\lambda$. Furthermore, the interacting Bose gas at $\lambda=2$ has the same scaled $\rho_{\infty}$ as the non-interacting free Fermi gas. This seems to suggest that not only the strength of the interaction but also the specific type of the interaction is irrelevant. We do not know whether this behavior may be explained in terms of the conformal symmetry of the system, but hope to come back to this point in a future publication.

Trying to classify the $g / r^{2}$ model by its critical anomaly, we calculated the finite-size corrections to the ground state energy as in (1). We found that in the cylindrical geometry, $c$ depends continuously on the value of the coupling constant $\lambda$. On the other hand, use of the low-temperature expansion of the free energy showed that $c=1$. Furthermore, the exponents $\alpha_{B}, \alpha_{F}$ vary continuously as functions of $\lambda$. In [6] this has been shown to be a typical phenomena of conformal field theories with anomaly $c=1$. In addition, Kawakami and Yang [14] demonstrated that $\beta_{B}, \beta_{F}$ in our model obey the scaling relations of the $c=1$ Luttinger liquid. Therefore, we believe that $c=1$ is the correct value of the critical anomaly of the $g / r^{2}$ model. We emphasize that for models with $c \geq 1$ there is no Kac-formula, relating the critical anomaly and the scaling dimensions, so we proceeded to calculate the correlation exponents independently of $c$.

We conclude this paper by noting that at present, we do not know a satisfactory rigorous explanation for the observed discrepancy in the estimate of $c$ in terms of conformal field theory. However, we believe this to be a remainder of the long-range character of the $g / r^{2}$ interaction, and in the light of our introductory remarks of section [f, it should not be too surprising if time correlations differ from space correlations in long-ranged models.

\section{ACKNOWLEDGMENTS}

R.A.R. would like to thank Carlton De Tar for helpful comments. 


\section{REFERENCES}

[1] I. Affleck, Phys. Rev. Lett. 56, 746, (1986).

[2] H. W. J. Blöte, J. L. Cardy, M. P. Nightingale, Phys. Re. Lett. 56, 742, (1986).

[3] A. A. Belavin, A. M. Polyakov, A. B. Zamolodchikov, Nuc. Phys. B 241, 333, (1984).

[4] J. L. Cardy, Nuc. Phys. B270 [FS16], 186, (1986).

[5] J. L. Cardy, Conformal Invariance in Phase Transitions and Critical Phenomena, eds. C. Domb, J. L. Lebowitz, vol. 11, Academic Press, (1987).

[6] J. L. Cardy, J. Phys. A: Math. Gen. 20, L891, (1987).

[7] Vl. S. Dotsenko, Nuc. Phys. B235 [FS16], 54, (1984).

[8] F. Dyson, J. Math. Phys. 3, 140, (1962).

[9] D. Friedan, Z. Qui, S. Shenker, Phys. Rev. Lett. 52, 1575, (1984).

[10] F. Gebhardt, D. Vollhardt, Phys. Rev. Lett. 59, 1472, (1987).

[11] M. Girardeau, J. Math. Phys. 1, 516, (1960).

[12] F. D. M. Haldane, Phys. Rev. Lett. 60, 635, (1988).

[13] F. D. M. Haldane, Phys. Rev. Lett. 66, 1529, (1991).

[14] N. Kawakami, S.-K. Yang, Phys. Rev. Lett. 67, 2493, (1991).

[15] E. B. Kolomeisky, preprint.

[16] A. Lenard, J. Math. Phys. 5, 930, (1964).

[17] M. L. Metha, Random Matrices, Academic Press, (1991).

[18] A. D. Mironov, A. V. Zabrodin, Phys. Rev. Lett. 66, 534, (1991).

[19] A. M. Polyakov, Zh. Eksp. Teor. Fiz. Pis. Red. 12, 538, (1970) [Sov. Phys. JETP Lett. 12, 381, (1970)].

[20] T. D. Schultz, J. Math. Phys. 4, 666, (1963).

[21] B. S. Shastry, Phys. Rev. Lett. 60, 639, (1988).

[22] B. Sutherland, J. Math. Phys. 12, 246 (1971); 12, 251 (1971); Phys. Rev. A 4, 2019, (1971); 5, 1372, (1972).

[23] B. Sutherland, Phys. Rev. B 45, 907, (1992).

[24] D. J. Thouless, Phys. Rev. 187, 32, (1969). 


\section{FIGURES}

FIG. 1. Correlation exponents for bosonic and fermionic systems as functions of the interaction strength $g$. The values corresponding to the special points $g=-\frac{1}{2}, 0,4$ are indicated.

FIG. 2. Momentum distributions for the bosonic system on a lattice at half-filling, for the special values of $\lambda$.

FIG. 3. Momentum distributions for the fermionic system on a lattice at half-filling, for the special values of $\lambda$.

FIG. 4. Estimates of the critical exponents as functions of the inverse length of the lattice. $(\square)$ corresponds to $\beta_{B, \frac{1}{2}},(+)$ to $\alpha_{F, 2}$ and $(\diamond)$ to $\alpha_{F, \frac{1}{2}}$.

FIG. 5. Scaled one-particle density $\rho_{\infty}$ for the bosonic system at half-filling, for the special values of $\lambda$. The curves for the discrete and the continuous case are identical up to the accuracy of the plot. The end points are normalized to 1 .

FIG. 6. Scaled one-particle density $\rho_{\infty}$ for the fermionic system at half-filling, for the special values of $\lambda$. The curves for the discrete and the continuous case are identical up to the accuracy

of the plot. The end points are normalized to 1 . Note that the curves for $\lambda=\frac{1}{2}$ and $\lambda=2$ are identical, too. 


\section{TABLES}

TABLE I. Results for the critical exponents $\alpha$ from conformal finite-size scaling analysis and numerical calculations for the special values of $\lambda$. The estimated error for the last digit is given in parenthesis. The $*$ indicates that the value of the corresponding exponent is known exactly.

\begin{tabular}{lccc}
\hline \hline & & & $\alpha$ \\
& $\lambda$ & Conformal & Numerical \\
& \multicolumn{1}{c}{ Pesults } \\
\hline \multirow{3}{*}{ Bose } & $\frac{1}{2}$ & Predictions & $-0.750002(5)$ \\
& $1^{*}$ & $-\frac{3}{4}$ & $-0.500000(1)$ \\
& $2^{*}$ & $-\frac{1}{2}$ & $0.0000000(1)$ \\
Fermi & $\frac{1}{2}$ & 0 & $0.25001(4)$ \\
& $1^{*}$ & $\frac{1}{4}$ & $0.0000000(1)$ \\
& 2 & 0 & $0.25000(1)$ \\
\hline \hline
\end{tabular}




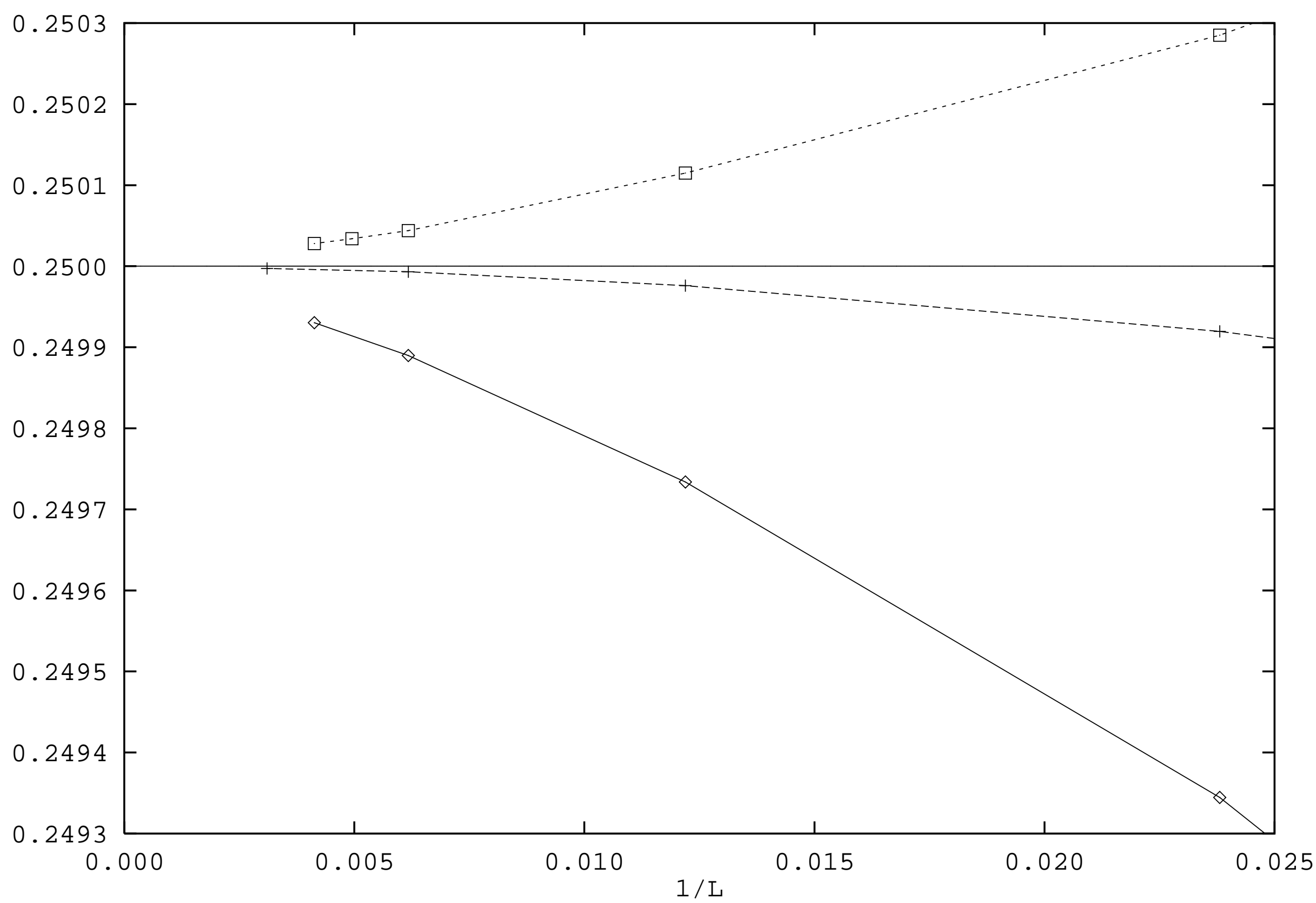




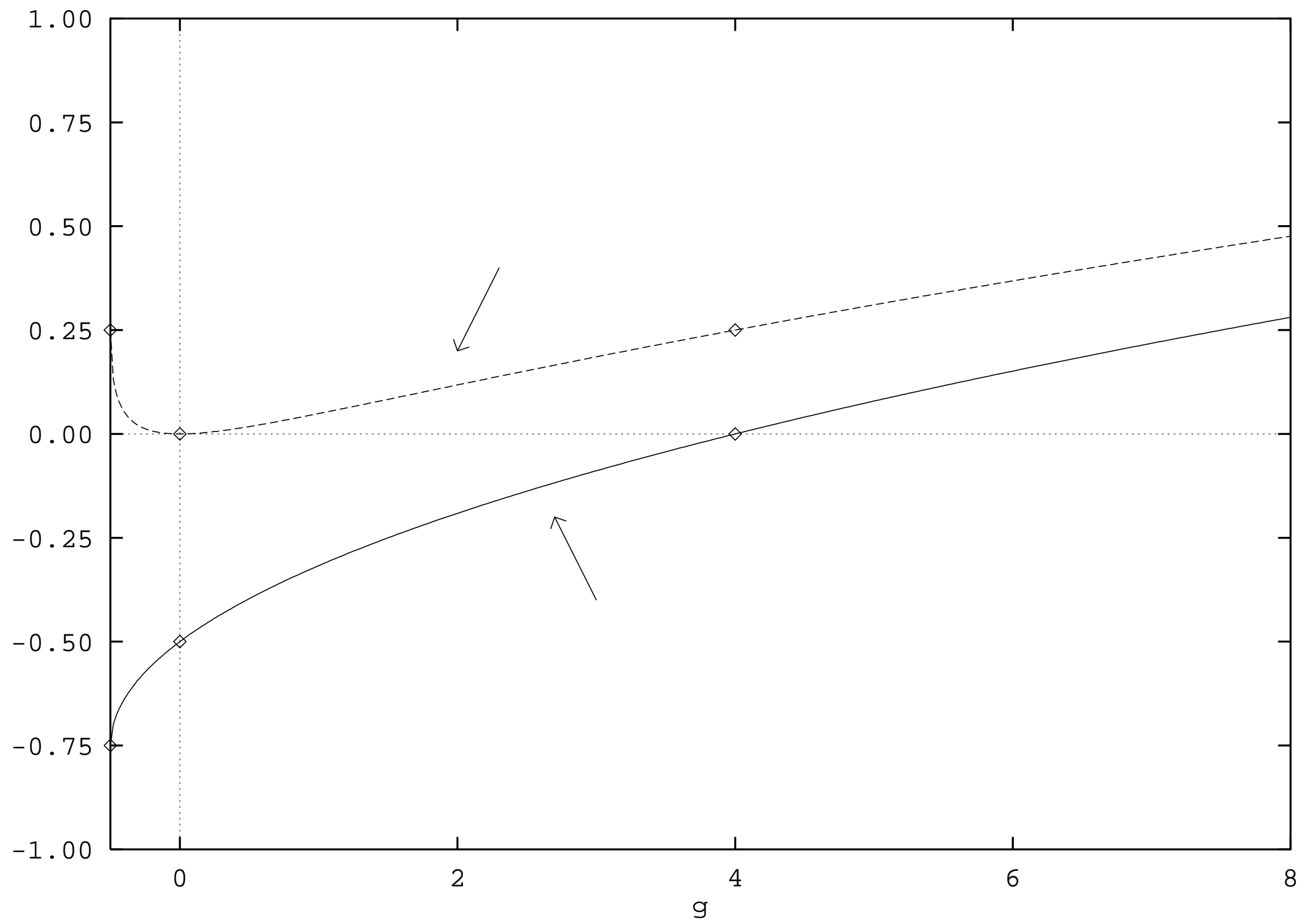




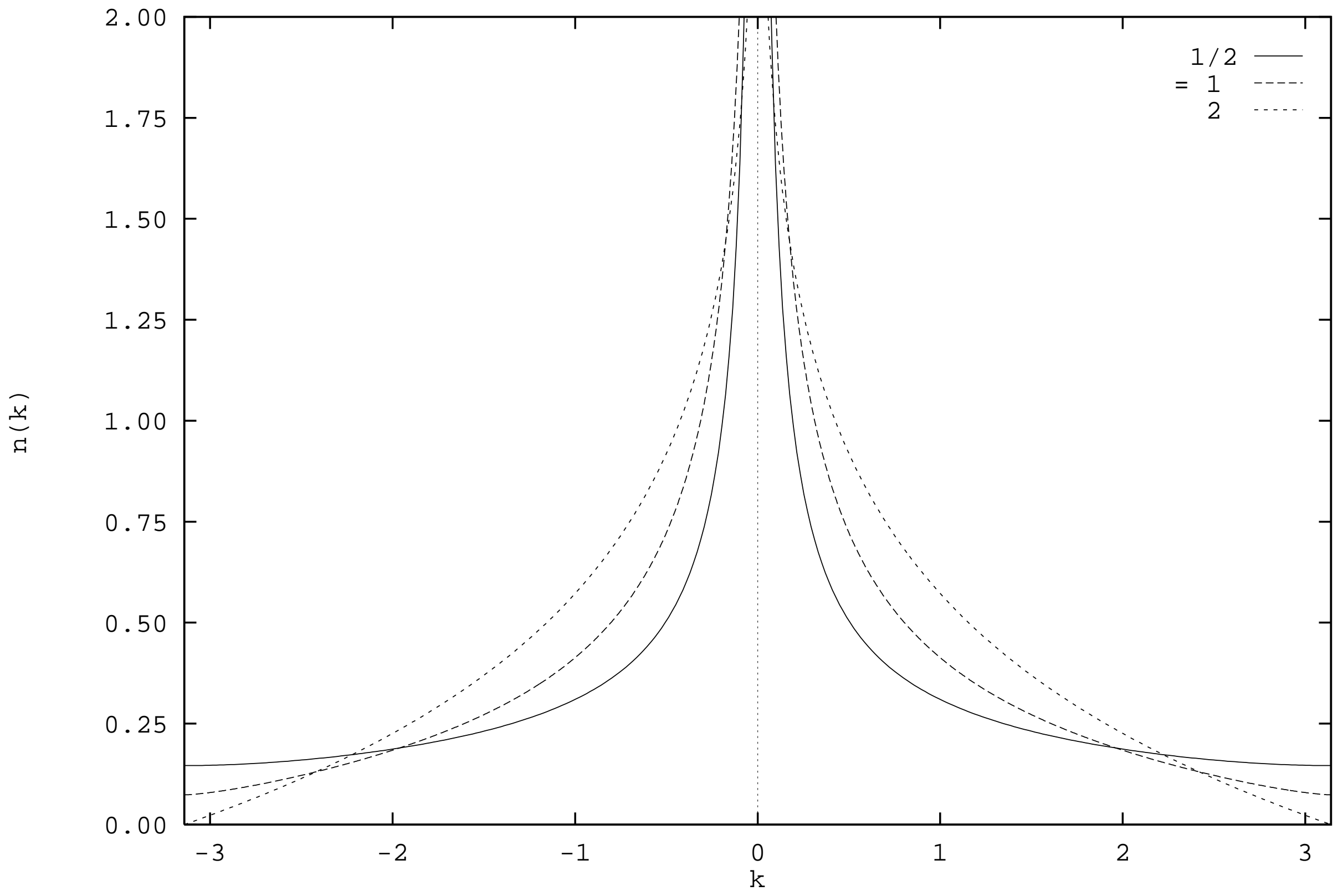




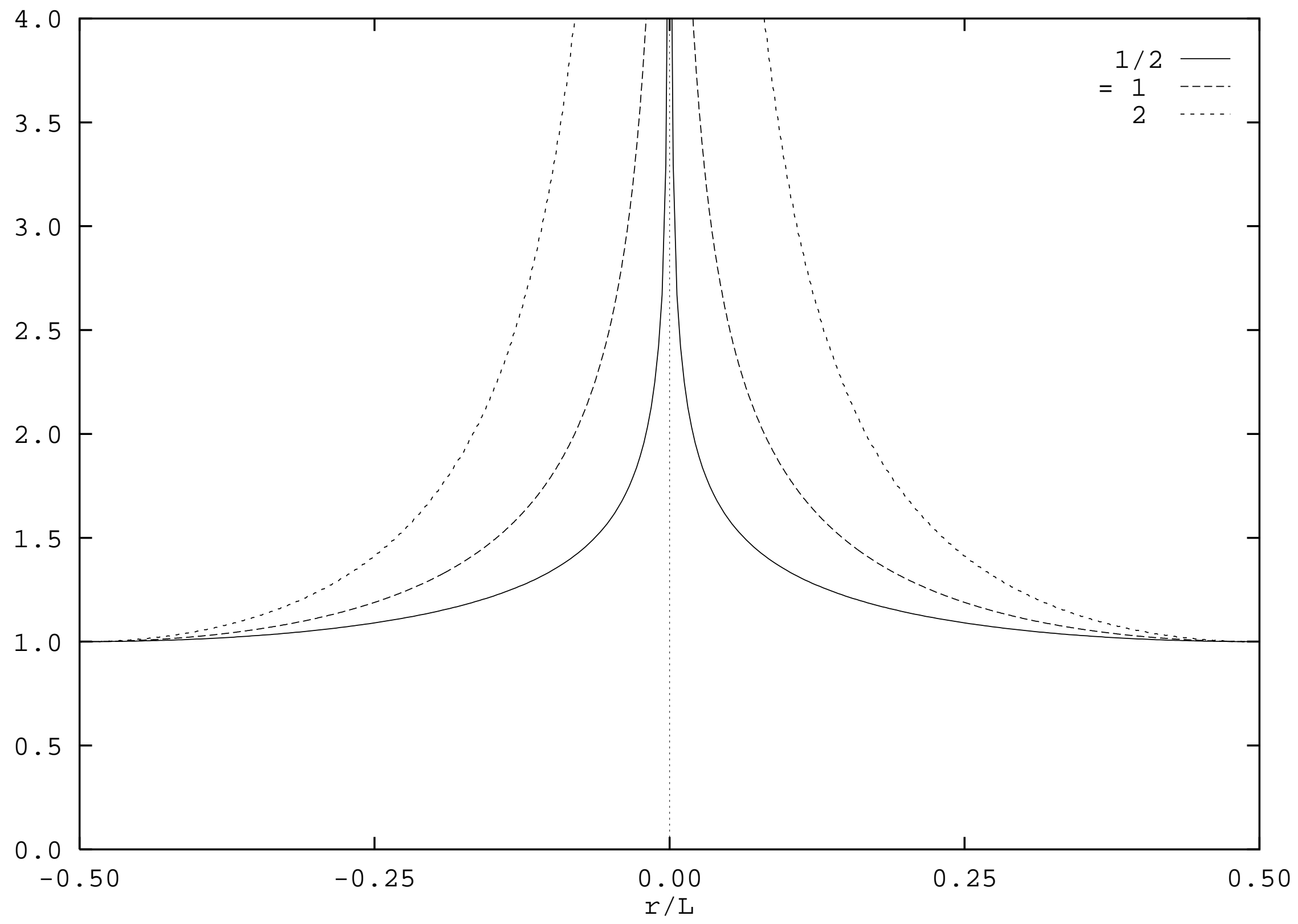




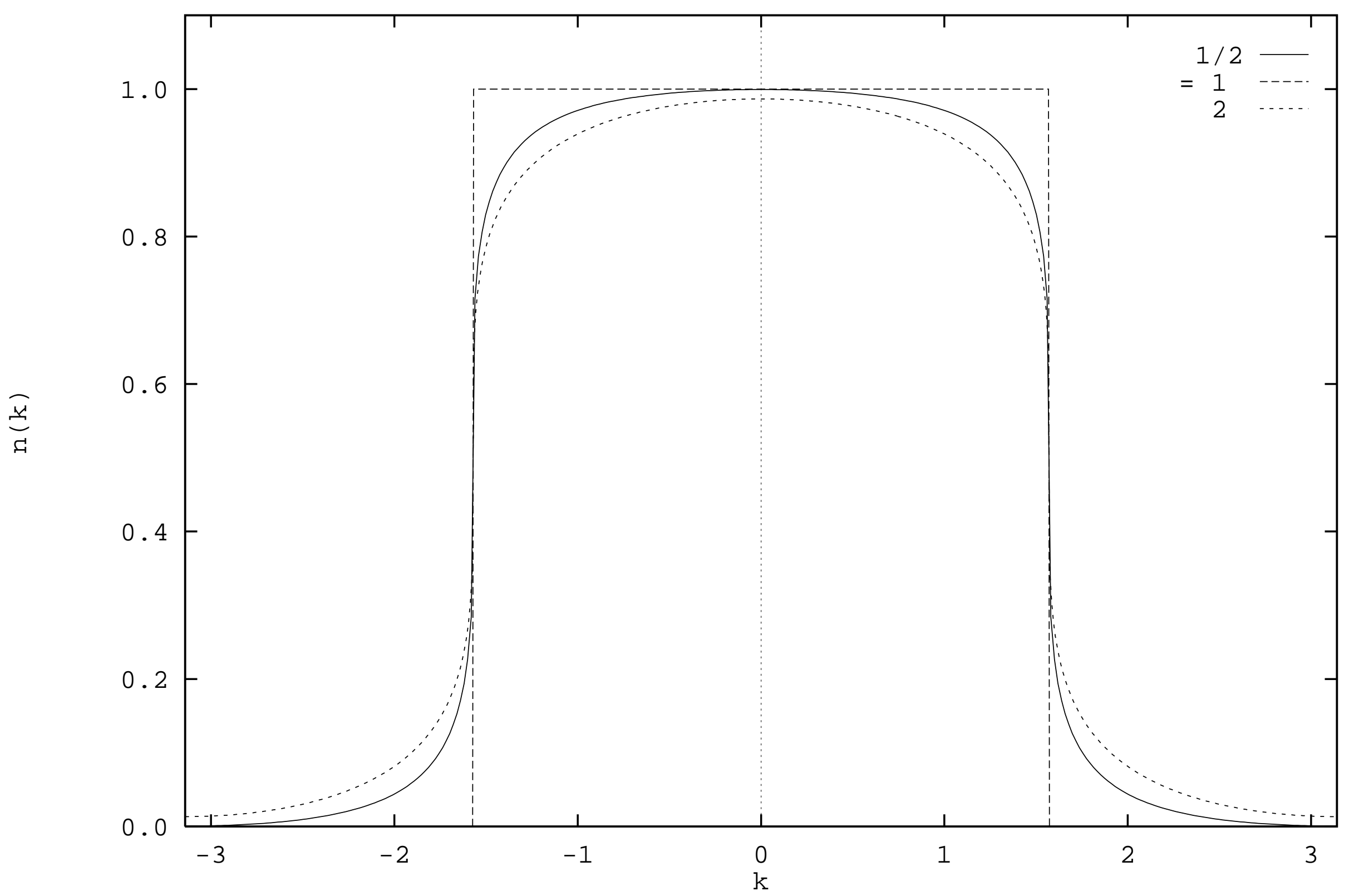




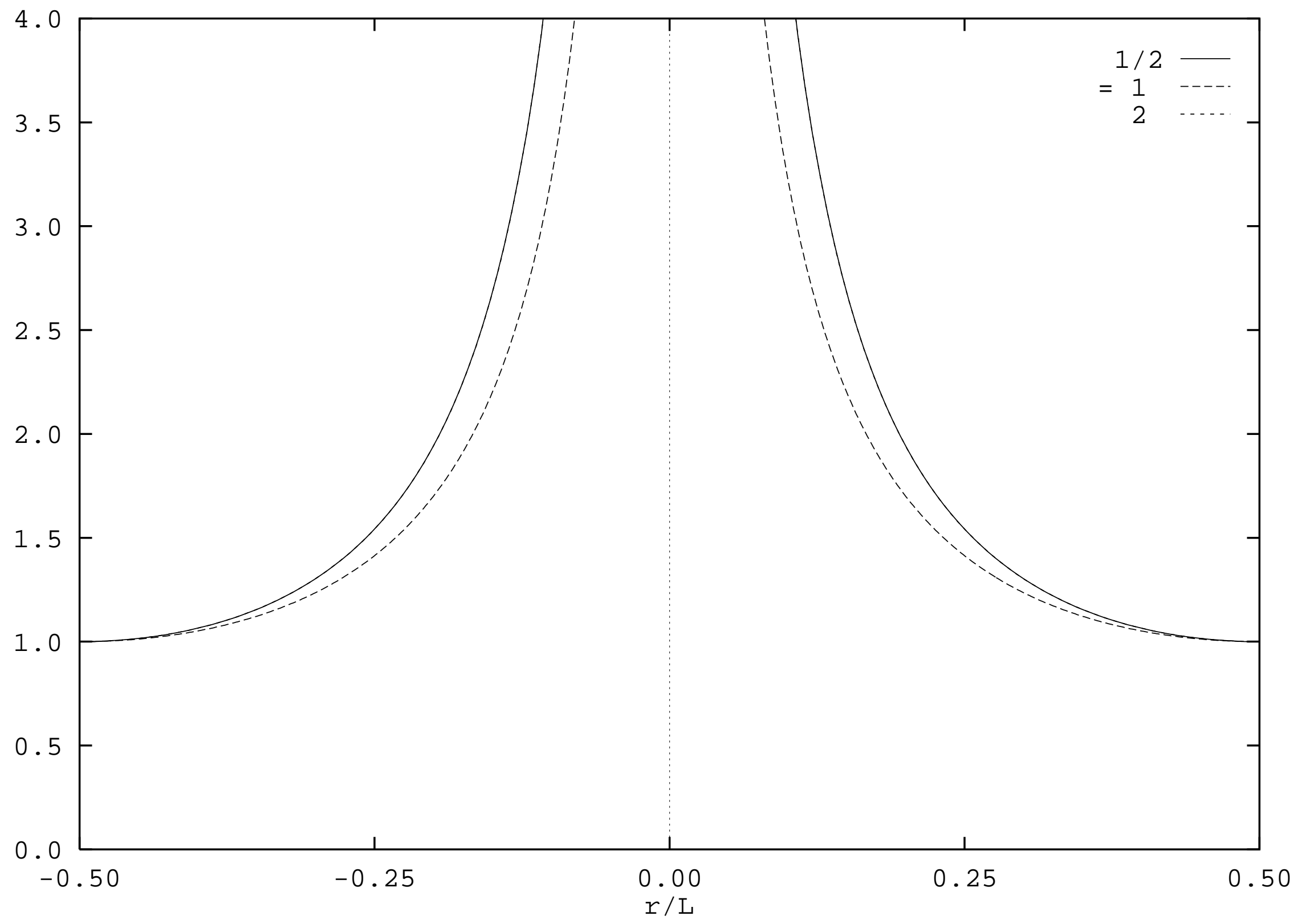

